\title{
The origin of observed magnetic variability for a sol on Mars from InSight
}

\author{
A. Mittelholz ${ }^{1}$, C. L. Johnson ${ }^{1,2}$, S. N. Thorne ${ }^{1}$, S. Joy ${ }^{3}$, E. Barrett ${ }^{4}$, M. \\ Fillingim $^{5}$, F. Forget ${ }^{6}$, B. Langlais ${ }^{7}$, C. T. Russell ${ }^{3}$, A. Spiga ${ }^{7,8}$, S. Smrekar ${ }^{4}$, \\ and W. B. Banerdt ${ }^{4}$ \\ ${ }^{1}$ Department of Earth, Ocean and Atmospheric Sciences, The University of British Columbia, Vancouver, \\ Canada. \\ ${ }^{2}$ Planetary Science Institute, Tucson, Arizona, USA. \\ ${ }^{3}$ Department of Earth, Planetary, and Space Sciences, University of California, Los Angeles, USA. \\ ${ }^{4}$ Jet Propulsion Laboratory, California Institute of Technology, Pasadena, USA. \\ ${ }^{5}$ Space Sciences Laboratory, University of California, Berkeley, USA. \\ ${ }^{6}$ Laboratoire de Météorologie Dynamique / Institut Pierre Simon Laplace, Sorbonne Université, Centre \\ National de la Recherche Scientifique (CNRS), École Polytechnique, École Normale Supérieure (ENS), \\ France. \\ ${ }^{7}$ Laboratoire de Planétologie et Géodynamique, UMR 6112, Université de Nantes, Université d'Angers, \\ CNRS, Nantes, France. \\ ${ }^{8}$ Institut Universitaire de France, Paris, France.
}

\section{Key Points:}

- InSight magnetometer data for sols 14 - 389 show daily magnetic field variations.

- The field strength consistently peaks in early-mid morning, with typical amplitudes 20 - $40 \mathrm{nT}$ but can reach almost $80 \mathrm{nT}$.

- Wind-driven ionospheric currents predict some of the observed signal, including the increased amplitude during a regional dust storm. 


\begin{abstract}
Day-night variations in the magnetic field at Mars have been previously observed at satellite altitudes. The InSight Fluxgate Magnetometer (IFG) has provided the first evidence for daily magnetic field variations at the martian surface. IFG data show diurnal variations with typical peak amplitudes of of $20-40 \mathrm{nT}$ in the early to mid morning; the amplitude of the magnetic field varies over the first 389 sols of the mission and peaks between sols 50 and 100. Temperature variations, solar array currents and lander activities all generate magnetic fields. Particularly, the first two of these produce signals with clear diurnal variations. We first assess the IFG data calibration and conclude that temperature and solar array currents have only minimal effects on the variability we observe in the final calibrated magnetic field data. We use satellite magnetic field data and a Mars Global Circulation Model to make predictions for the temporal evolution of wind-driven fields in the ionosphere. Such fields vary due to seasonal changes in the ionization profile and the winds, and in the altitude range of the dynamo region, i.e. the region in which electric currents can be produced. We find that the amplitude and seasonal variability of the surface magnetic fields are generally consistent with those predicted from winddriven currents. Moreover, a regional dust storm in the vicinity of the InSight landing site, that started around sol 45, might be responsible for the higher magnetic field amplitudes observed in the IFG data in the early part of the mission.
\end{abstract}

\title{
Plain Language Summary
}

The InSight lander carries a magnetometer, that has for the first time measured magnetic fields on the surface of Mars. The observed magnetic field strength varies from day to night and peaks in the early-to-mid morning. We report how the diurnal variations have evolved over the first 389 sols (martian days) of the InSight mission, and show that there are substantial sol-to-sol changes as well as more gradual changes over longer timescales. We investigate whether these are caused by the diurnal variations in temperature of the magnetometer instrument and the electronics box, day-night-variations in the currents drawn from the lander's solar arrays, and lander activities such as movement of the arm and satellite communications. We conclude that these sources are generally well accounted for in the processed magnetometer data. We then investigate whether the daily variations in the field could be caused by charged particles in the upper atmosphere (ionosphere) that move with the wind and generate electric currents. We model this process using a climate model for Mars and find that the predicted magnetic fields are consistent with the observed evolving diurnal pattern. This indicates that at least some of the magnetic field variability on ground is indeed generated in the ionosphere.

\section{Introduction}

InSight, the Interior Exploration using Seismic Investigations, Geodesy and Heat Transport mission, landed on Mars in November 2018 with the primary goal of studying the deep interior of Mars (Banerdt et al., 2020). The main science payload includes a seismometer, a heat flow probe and a radio antenna. Several auxiliary instruments were included to monitor environmental conditions that could be detected by the seismometer. One of those, the InSight Fluxgate Magnetometer (IFG) is providing the first magnetic field measurements from the surface of Mars and has been operating almost continuously since sol 14 (Johnson et al., 2020).

Magnetic field data recorded by the IFG have revealed a static crustal magnetic field with an amplitude of $2013 \pm 53 \mathrm{nT}$, much stronger than the surface field strength predicted from models based on satellite observations (Smrekar et al., 2018; Johnson et al., 2020; Banerdt et al., 2020). Furthermore, IFG data show time-varying fields (Johnson et al., 2020) including variations at the daily period, i.e., the period of one martian sol, and its harmonics, magnetic 'pulsations' with frequencies of $\sim 100$ seconds to $\sim 10$ min- 
utes that occur on some sols at specific local times (Chi et al., 2019), and transient signals, many of which are of lander and not martian origin.

Here we examine the daily variations in the martian surface magnetic field. These can result from electric currents in the ionosphere that are driven by atmospheric winds between $\sim 130 \mathrm{~km}$ and $\sim 180 \mathrm{~km}$ altitude, in the so-called dynamo region. In this region, ions are collisionally coupled to neutral winds, while electrons gyrate about field lines. The differential motion of ions and electrons builds up ionospheric currents. The heights at which electrons or ions are no longer coupled to neutral winds are given by $h_{\mathrm{e}}=\nu_{\mathrm{en}} m_{\mathrm{e}} /\left|q_{\mathrm{e}}\right| B$ and $h_{\mathrm{i}}=\nu_{\mathrm{in}} m_{\mathrm{i}} /\left|q_{\mathrm{i}}\right| B$ respectively, where $m_{\mathrm{i}}, m_{\mathrm{e}}$ are the ion and electron masses, $B$ is the ambient magnetic field strength and $\nu_{i n}, \nu_{e n}$ are the ion-neutral and electron-neutral collision frequencies. At a given altitude above the surface on Mars, both $\nu_{\text {in }}, \nu_{\text {en }}$, as well as $B$, are lower than on the Earth, because of the thinner atmosphere and the absence of a global magnetic field (Opgenoorth et al., 2010). However, the difference in the magnetic fields between the two planets is much larger than the differences in collision frequency, and thus the dynamo region on Mars is at higher altitudes than on Earth (Opgenoorth et al., 2010). As a consequence, the dynamo region on Mars overlaps a region of higher plasma density, whereas on Earth it lies in a region of higher neutral densities, well below the peak ionization altitudes.

Several previous studies have attempted to predict the magnitude and variability of ionospheric currents and the resulting magnetic field, using satellite observations, as well as ionospheric, magnetohydrodynamic (MHD) and Mars Global Climate Models (MGCM). Withers et al. (2005) analytically calculated the expected altitude range of the dynamo region, as well as the electrical conductivities and current densities in that region and the resulting magnetic fields. They assumed a background magnetic field strength of 100 $\mathrm{nT}$ (appropriate to regions of moderate-strength crustal fields), a constant electron number density, $n_{\mathrm{e}}$, across the dynamo region and a horizontal wind speed of $100 \mathrm{~ms}^{-1}$. The resulting current densities, $j$, were $\sim 1 \mu \mathrm{Am}^{-2}$ producing a magnetic field of $\sim 40 \mathrm{nT}$ at ionospheric altitudes. Further, it was shown that the presence of strong crustal magnetic fields influences the electrical conductivity structure. Specifically, weak crustal magnetization was shown to enhance conductivity especially at altitudes around $130 \mathrm{~km}$ (Opgenoorth et al., 2010). Other studies using different approaches and assumptions also predicted the magnitude of neutral-wind-driven ionospheric currents to be $\sim 1 \mu \mathrm{Am}^{-2}$ (Fillingim et al., 2010, 2012; Riousset et al., 2014; Lillis et al., 2019), approximately two orders of magnitude greater than currents generated in nondynamic conditions, e.g., driven by plasma pressure gradients (Fillingim et al., 2010; Withers et al., 2005; Riousset et al., 2014; Lillis et al., 2019). Mittelholz et al. (2017) assessed the average diurnal signal of the external (non-crustal) magnetic field as observed by the Mars Global Surveyor (MGS) satellite at around $400 \mathrm{~km}$ altitude, i.e., well above the ionospheric peak. They found that the observed magnitude of $\sim 10 \mathrm{nT}$ at MGS altitudes and the zonal structure of the averaged field could be explained by wind-driven currents in the ionosphere. This is consistent with the work of Fillingim et al. (2012) who predicted ionospheric contributions of $\sim 10 \mathrm{nT}$ at the surface and $\sim 50 \mathrm{nT}$ at $150 \mathrm{~km}$. A recent study by Lillis et al. (2019) specifically focused on the predicted surface field at the InSight landing site using atmospheric conditions from a MGCM and the ambient magnetic field from a MHD model assuming a constant interplanetary magnetic field (IMF) for the duration of the MHD simulation. Predicted field strengths at the surface were $10-100 \mathrm{nT}$, depending on season and therefore on $n_{\mathrm{e}}$ and wind direction. A further complication is that in some regions crustal fields are sufficiently strong that they modify the ambient field direction in the ionosphere, thereby influencing the currents and resulting fields that are generated (Riousset et al., 2014).

From this, we conclude that daily magnetic field variations at the surface from ionospheric currents (with $j \approx 1-10 \mu \mathrm{Am}^{-2}$ ) are expected with amplitudes of a few nT up to $\sim 100 \mathrm{nT}$. The magnitude likely varies with time because conditions in the ionosphere 
change; $n_{\mathrm{e}}$ and the winds will vary with season and local time (Withers et al., 2015; Zou et al., 2011), and the IMF varies with a 26-day cycle corresponding to Carrington rotations, i.e., the solar synodic rotation period at Mars, as well as on shorter periods (Marquette et al., 2018). A priori, we thus expect daily variations in the magnetic field that may also have aperiodic, seasonal and 26-day (and harmonics thereof) modulations. Such modulations were observed in MGS magnetic field measurements (Langlais et al., 2017; Mittelholz et al., 2017). IFG data at the inSight landing site provide the first surface constraints on the daily variations in the magnetic field, and these can be compared with model predictions to provide new constraints on ionospheric processes.

Analyses of the diurnal magnetic field variations recorded by the IFG require consideration of variations that are not of natural origin. Specifically, these include diurnal temperature variations that affect the gain of the magnetometer, solar array currents that are present during the daytime, as well as day-night variations in the occurrence rate of lander operations, such as movement of the robotic arm or communication (Thorne et al., 2020; Johnson et al., 2020; Banerdt et al., 2020). In fluxgate magnetometers such as the IFG, ambient T changes affect the windings of the sensor coils and/or the feedback coils and hence the resulting measured magnetic field. The ambient temperature at the sensor head is measured directly. In addition to the winding geometry changes, there can be changes in the magnetic permeability of the sensor mu metal core with large temperature variations (Miles et al., 2017).

In this paper, we first show the evolution of diurnal magnetic field variations over 376 sols on Mars, spanning the time period from the beginning of the InSight mission up to December 31, 2019 (Section 2). We then assess the temperature and solar array calibrations used in processing IFG data, to determine the extent to which diurnal signals in the calibrated IFG data might still contain contributions that are not of martian magnetic origin (Section 3 and Supplementary Information). We use an MGCM (Forget et al., 1999; González-Galindo et al., 2013) to predict neutral density profiles, electron density profiles and neutral winds as a function of altitude, local time and season above the InSight landing site. We also investigate how these quantities can vary during dust season. We take magnetic field data from MAVEN and MGS, as well as models for the crustal magnetic field, to characterize the altitude-dependence of the magnetic field, and combine this information with the outputs of the MGCM to assess how the lower altitude of the dynamo region varies seasonally and during times of dust. We then compute the electric current densities in the resulting dynamo region. Such currents produce magnetic fields; we estimate the resulting magnetic field at ionospheric altitudes and scale to predict the corresponding surface field strength. Finally, we compare such wind driven magnetic fields with InSight IFG predictions to date (Section 4).

\section{The Evolution of the Magnetic Field over Time}

\subsection{Data}

We select IFG data from the beginning of continuous IFG operations on sol 14 until sol 389 (Figure 1a), all publicly available as V4 data on the Planetary Data System (PDS) (Joy et al., 2019). We use $0.2 \mathrm{~Hz}$ data whenever this is the continuous data rate (sols 14-182 and sols 261-269). For time spans where $2 \mathrm{~Hz}$ data are continuously available (sols 182-261 and since sol 284), we use $2 \mathrm{~Hz}$ data downsampled on the ground to $0.2 \mathrm{~Hz}$ (labeled as gpt2 on the PDS). The coordinate system is the Lander Level frame (Joy et al., 2019), a local reference frame akin to that used in geomagnetism, in which $\hat{\mathrm{x}}$ is north, $\hat{\mathrm{y}}$ is east and $\hat{z}$ is down. Gaps occurred during Payload Auxiliary Electronics (PAE) anomalies and also between sols 270 and 283, when InSight experienced an anomaly that could not be diagnosed and fixed until after communication with the spacecraft resumed following the end of solar conjunction. 

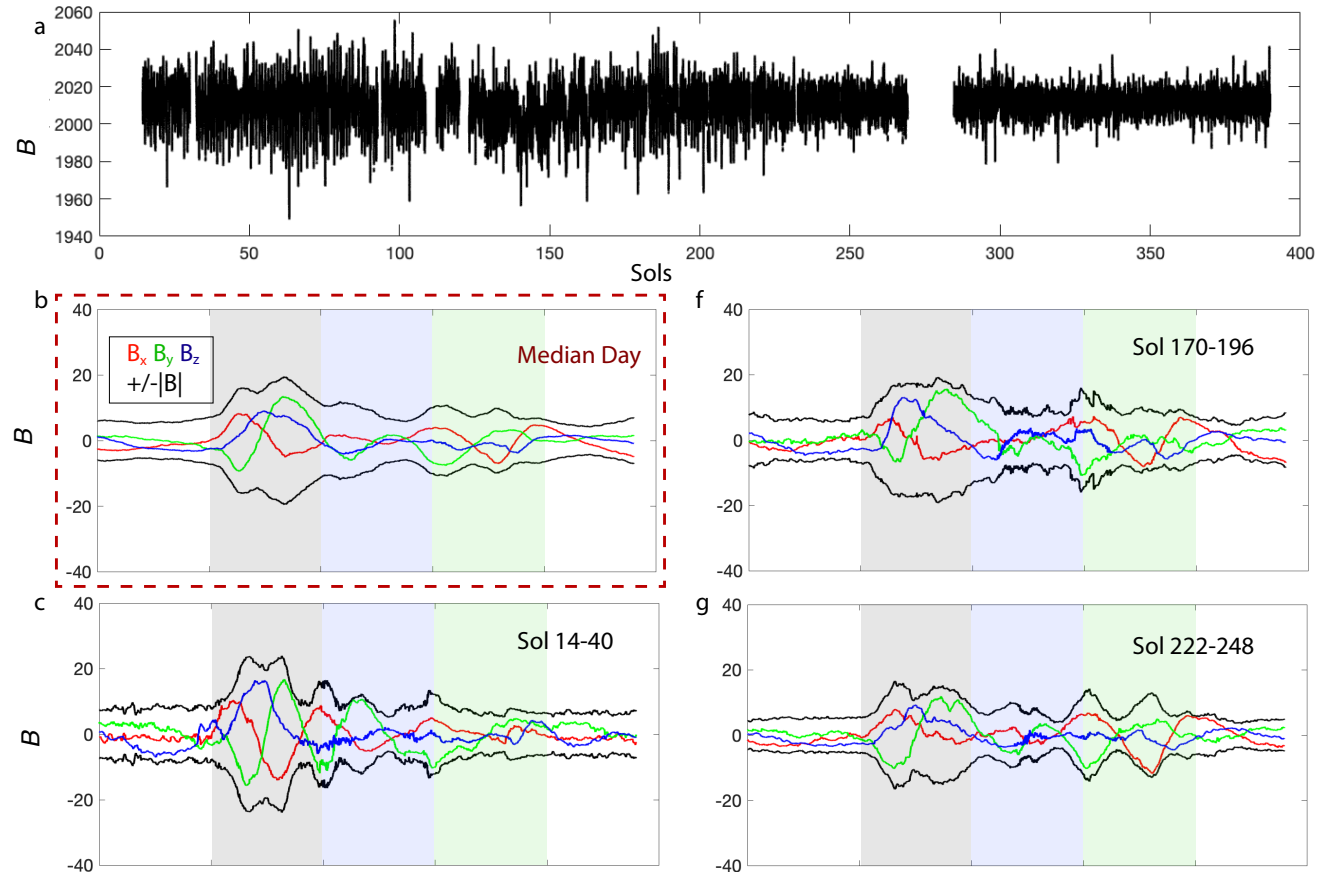

$\infty$
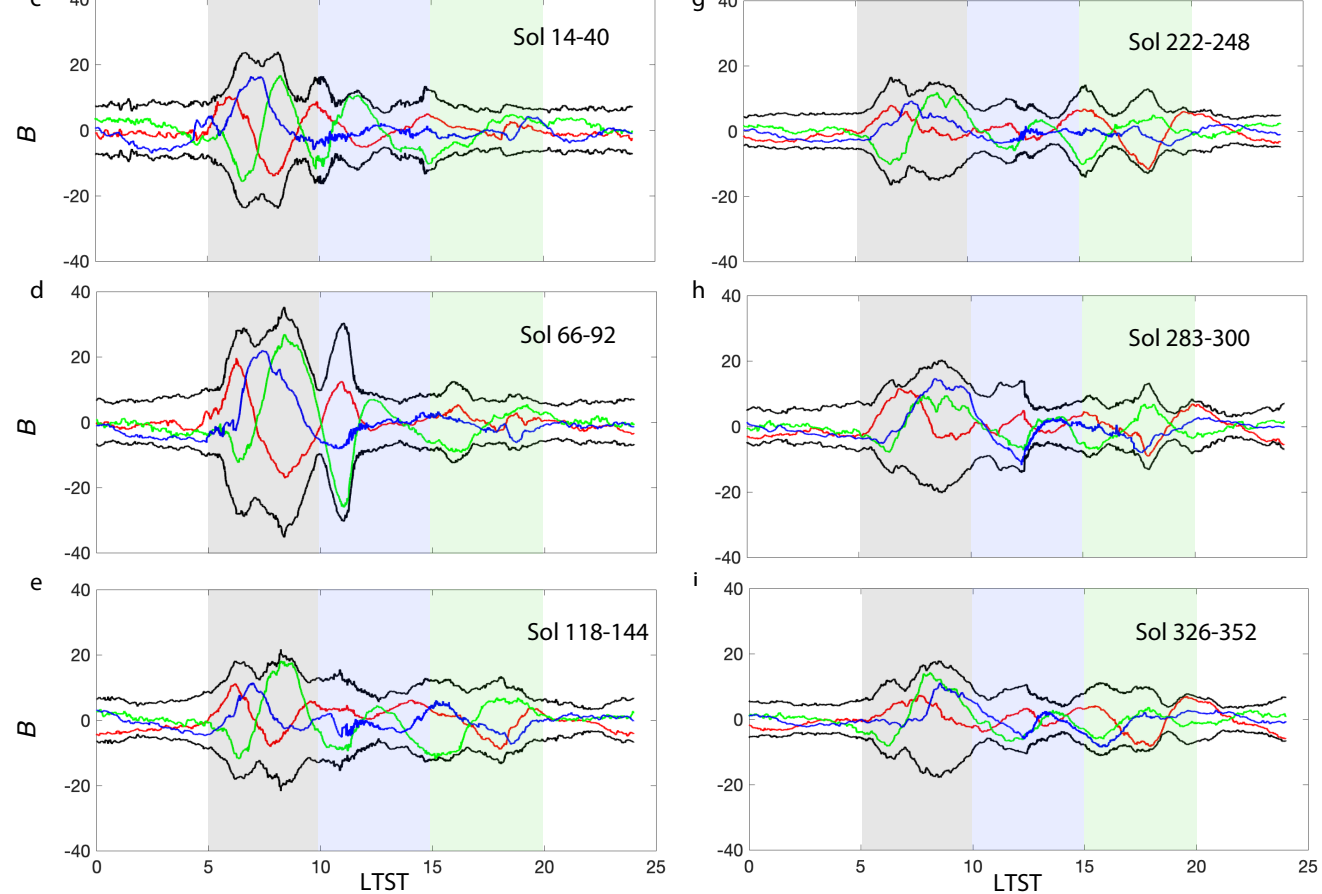

Figure 1. (a) Magnetic field strength, B, for sols 14-389. (b) The median daily signal over sols 14-389 as a function of local true solar time (LTST) for each component, in 10000 local time bins. $B_{x}$ is red, $B_{y}$ is green, $B_{z}$ is blue, $|\mathrm{B}|$ is black, with the mirror image of $B$ (i.e., $-B$ ) also shown so that time intervals in which any one component dominates the signal can be easily identified. (c)-(i) Median signal over 26-sol intervals, shown for alternating 26-sol time periods to cover the discussed time frame. The shaded grey, blue and green regions highlight local times from $05.00 \mathrm{hr}-10.00 \mathrm{hr}, 10.00 \mathrm{hr}-15.00 \mathrm{hr}$, and $15.00 \mathrm{hr}-20.00 \mathrm{hr}$. 


\subsection{Magnetic Field Observations: Diurnal Variations}

IFG data exhibit clear temporal variations (Figure 1a). Detailed inspection of the time series suggests that these are diurnal in nature, and this was confirmed by spectral peaks at the daily period and its harmonics (Johnson et al., 2020). The longer time series now available shows an overall decrease in amplitude over the almost 400 sols of data (Fig. 1a). We examine the diurnal variation and its evolution as follows. First, we compute the median signal as a function of local true solar time (LTST) using all data between sol 14 and 389. We detrend the data by removing the linear trend to remove the background crustal field and any long time-scale variation, bin the data into 10000 LTST bins corresponding to $\sim 9$ seconds per bin and take the median in each local time bin (Figure 1b). The resulting signal is small at night, with the largest amplitude signals occurring in the morning between $05.00 \mathrm{hr}$ and $10.00 \mathrm{hr}$ LTST. Individual sols sometimes exhibit high-frequency variations (not shown); however these are often correlated with specific lander operations (see extended data Figure 5 of Johnson et al. (2020)) and are not the focus of this study. We prefer the median over the mean so that such outliers do not bias the diurnal signature.

Motivated by these observations we examine the evolution of the median diurnal signal on 26-sol timescales. For each 26-sol time frame, we remove sols that contain data gaps longer than 10 minutes. For time frames that contain more than $50 \%$ of the sols (i.e., 13 days), we detrend the data by subtracting the linear trend of the time frame, bin and compute the median as a function of LTST as for the complete time series. We choose 26-sol as our time interval (i.e., the approximate time frame of a Carrington rotation) to ensure sufficient statistics for obtaining a reliable estimate of the 'typical' diurnal signal over a fairly short time period. At the same time a 26 -sol window is short enough so that we can capture seasonal variations and the effects related to the Carrington rotation should be averaged out. The 26 -sol median signals show some consistent structure (Figures 1b-i). The largest amplitude fields always occur in the early-to-mid morning. Earlier sols in general show a pronounced early morning peak up to almost 40 $\mathrm{nT}$, that for later sols does not exceed $20 \mathrm{nT}$. An increase in magnitude is also seen in the 10.00-15.00 hr interval, but its structure changes through the mission. The very pronounced peak just past $10.00 \mathrm{hr}$ (e.g., Figure 1c, d) that is seen earlier in the mission, vanishes at later sols, but a peak just before $15.00 \mathrm{hr}$ develops with maximum amplitude around sol 200. Evening and nighttime data exhibit mostly low amplitude signals. 
a

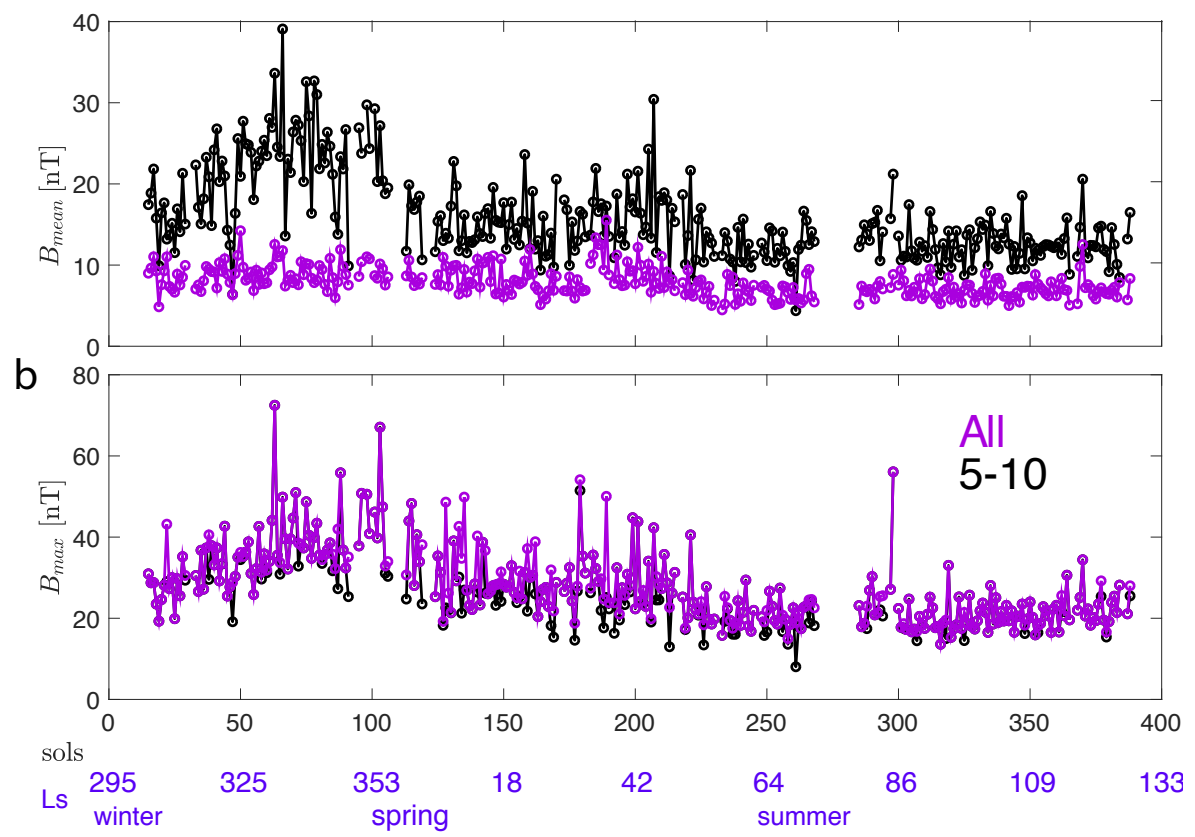

Figure 2. The (a) mean and (b) peak amplitude of the magnetic field (circles) per sol (i.e., no running window) to highlight the day-to-day variability for all LTST (purple), and LTST time intervals $05.00 \mathrm{hr}-10.00 \mathrm{hr}$ (black).

Furthermore, we evaluate the evolution of the daily mean and peak signal on a solby-sol basis (Figure 2) and for 26-sol windows (Figure 3). We also follow this procedure using subsets of data limited in local time. Sol-to-sol variability (Figure 2) can be quite large, with peak amplitudes that range from $20 \mathrm{nT}$ up to almost $80 \mathrm{nT}$ (Figure 2b), occurring almost always in the early morning. The mean daily signal is just below $\sim 10 \mathrm{nT}$ over the entire sol and $\sim 15-25 \mathrm{nT}$ for the 05.00 to $10.00 \mathrm{hr}$ period. Longer time-scale variations are also seen, and are more clearly evident in the mean and peak amplitudes computed from 26-sol windows (Figure 3). For the 26-sol windows, we only calculate the mean or peak daily value if at least 13 sols are available after removal of sols with data gaps longer than 10 minutes. The steep decrease in the mean or peak amplitude after sol 100 is mostly caused by the decrease in amplitude of the signal between $5.00 \mathrm{hr}$ and 10.00 $\mathrm{hr}$ (Figure 1). The evolution of the peak amplitude in the $10.00-15.00 \mathrm{hr}$ interval shows a similar pattern but with a smoother change around sol 100. In addition an increased amplitude just before sol 200 is seen. The change in mean amplitude for all local times is only a few $\mathrm{nT}$, in the early morning the mean ranges from $\sim 10 \mathrm{nT}$ up to about $25 \mathrm{nT}$.

We investigated whether there was a characteristic correlation time scale for the daily magnetic field variations by cross-correlating each complete sol with all other sols. A characteristic time scale would be evidenced by e.g. several successive days of high correlation coefficients, followed by a drop in correlation. We found that the correlation coefficient for the magnetic field strength was nearly always above 0.8 and did not show times of higher correlation compared to others, i.e., no characteristic time scale was found. This likely indicates that there is the same general diurnal variation in magnetic field strength, even if there is no evidence that it repeats in a detailed fashion for multiple days at a time.

We note that a time variable contribution is expected from the magnetization induced in the crust from inducing ionospheric magnetic fields. Although these two are 
coupled, calculations assuming an inducing field of maximum amplitude $100 \mathrm{nT}$ and susceptibility values reported in Rochette et al. (2005) indicate that induced magnetization is a very minor contribution to the total magnetization (i.e., $<1 \%$ ) at the InSight landing site (Johnson et al., 2020). Hence, we can disregard such induced contributions to the measured time-varying magnetic field in this study.
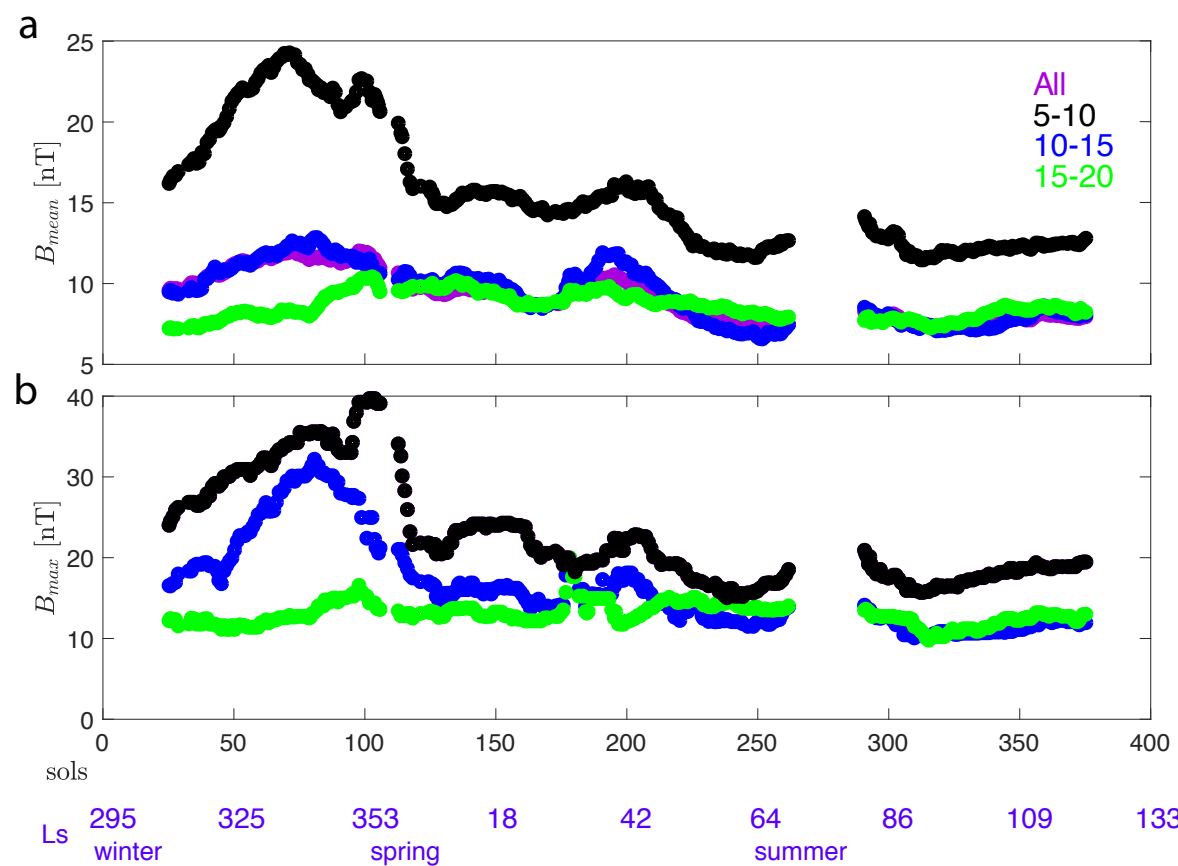

Figure 3. The (a) mean and (b) peak amplitude of the magnetic field (circles) using a 26-sol running window of the binned days for all LTST (purple), and LTST time intervals $05.00 \mathrm{hr}$ $10.00 \mathrm{hr}$ (black), $10.00 \mathrm{hr}-15.00 \mathrm{hr}$ blue), and $15.00 \mathrm{hr}-20.00 \mathrm{hr}$ (green). The mean and peak amplitude is plotted at the midpoint of the 26-sol window. Because the peak amplitude occurs between $05.00 \mathrm{hr}$ and $10.00 \mathrm{hr}$ the black and red symbols in (b) overly each other.

\section{Artificial Drivers of Variations}

As mentioned earlier the magnetometer was included in the InSight instrument payload for the purpose of characterizing magnetic signals of any origin (i.e., artificial or natural). Consequently spacecraft magnetic cleanliness was not required, and pre-launch characterization of magnetic sources was limited to two tests to evaluate the static magnetic moment of the lander itself, thermal calibration of the IFG from $\sim-60^{\circ} \mathrm{C}$ to room temperature, and a restricted set of tests regarding time-varying fields arising from specific spacecraft/commanding operations (Banfield et al., 2018; Joy et al., 2019).

For a full description of the calibration procedure and all raw calibration data we refer to the SIS and Calibration Documentation available on the PDS (Joy et al., 2019). In brief, the procedure involves steps (1)-(3) as follows: (1) Pre-launch calibrations of the IFG dependence on sensor and electronics temperature are used to convert raw IFG data from digital units into nT. (2) The pre-flight-determined spacecraft static field contribution is subtracted ([549, -434, 26.5] $\mathrm{nT}$ in the spacecraft frame). (3) Residual contributions from temperature and solar array currents are estimated via a linear least square fit to the magnetic field at the end of step (2). Although temperature calibrations were performed pre-launch, they did not encompass the full range of nighttime temperatures 
expected at the InSight landing site and thus residual, uncorrected temperature-related signals were anticipated in the IFG data. The fit is expressed as $\mathrm{dB}_{f i t}=c_{0}+c_{1} \mathrm{ST}+$ $c_{2} \mathrm{ET}+c_{3} \mathrm{SAC}+c_{4} \mathrm{SACT}$, where $c_{0}, c_{1}, c_{2}, c_{3}, c_{4}$ are the constants determined by the fit which is done in the IFG frame. The typical variations of the sensor temperature (ST), electronics temperature (ET), fixed solar array currents (SAC) and 'total' solar array currents (SACT) with local time are shown in Figure 4(a-d) and are described further in the supplementary information. The fits are performed individually for each magnetic field component and subtracted from the step 2 data.

The ST, ET, SAC and SACT data are provided at different sampling rates, and furthermore the SAC and SACT data are not continuous in time, so each of these data streams are interpolated or modeled at the IFG data rate. Relevant issues are thus how well the models for ST, ET, SAC, SACT describe the actual calibration data, and what their influence is on the final magnetic field data product.

In the supplementary information, we discuss the four data channels used in the calibration, the uncertainties associated with them, and the resulting corrections to the data. We also summarize the effects of transient lander signals. As a result of these various corrections, our conclusion is that while we cannot rule out some remaining contributions of thermal effects, solar array currents and lander activities to the signals shown in Figures 1-3, we believe any remaining contributions to be small and not responsible for the temporal evolution we see in the daily variation.

\section{Wind-driven Ionospheric Currents}

Wind-driven magnetic fields depend on atmospheric conditions, i.e., electron number density and winds, as well as the geometry and amplitude of the background magnetic field. All these quantities are dynamic and continuous observations at a given altitude and geographic location are not available. MAVEN (Jakosky et al., 2015) provides measurements of the magnetic field in the ionosphere that are intermittent in time and space because of its precessing, elliptical orbit. These data, can however be used to build a statistical picture of the magnetic field at ionospheric altitudes. Winds and electron number density are variable, but have seasonal variations that can be modeled using a MGCM. Furthermore, the altitude range of the dynamo region itself is dynamic and varies with factors such as neutral density, electron temperature and the background magnetic field.

In the following, we discuss the magnetic field (Section 4.1) and atmospheric properties (Section 4.2) in the dynamo region. We estimate the seasonal variations in the altitudes of the lower boundary of the dynamo region (Section 4.3). We compute the peak daily current-densities in the dynamo region, the resulting surface magnetic field strength and compare the magnitude and temporal variations in this strength with the InSight IFG data (Section 4.4).

\subsection{Magnetic Field at Ionospheric Altitudes}

The background magnetic field depends on both the static crustal field and the locally draped IMF. The contribution from the crustal field can be estimated using crustal field models built with MAVEN and Mars Global Surveyor (MGS) data. These data sets provide good coverage of ionospheric orbital altitudes down to about $\sim 135 \mathrm{~km}$ (Mittelholz et al., 2018; Langlais et al., 2019) and the field models at lower altitudes are downward continued. They predict crustal magnetic field amplitudes around 40-70 nT within the dynamo region (Figure 5), somewhat larger than the predictions of 20-30 nT, from earlier models based only on MGS data (e.g., Morschhauser et al. (2014)). 

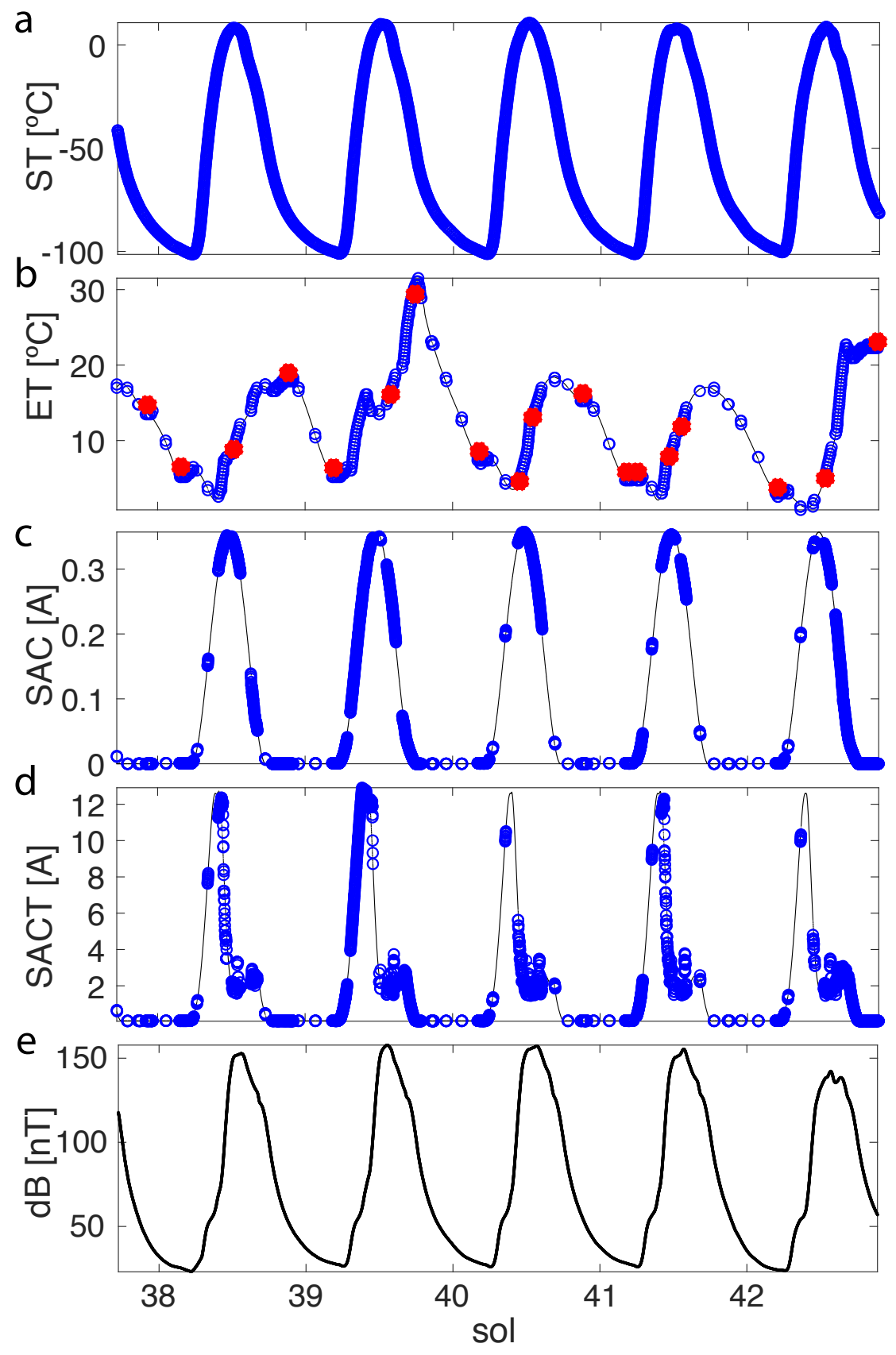

Figure 4. (a-d) Five representative sols of (a) sensor temperature, ST, (b) electronics temperature, ET, (c) fixed solar array current data, SAC, and (d) total solar array current data, SACT showing the models used for calibration (black solid line) and the data on which the models are based (blue circles). For ET we also show the actual ET (red stars), but the model was built using a proxy (blue circles) due to limited ET availability until sol 347. (e) The amplitude of the $\mathrm{dB}$ fit resulting from step (3) (see supplementary info for more explanation). 


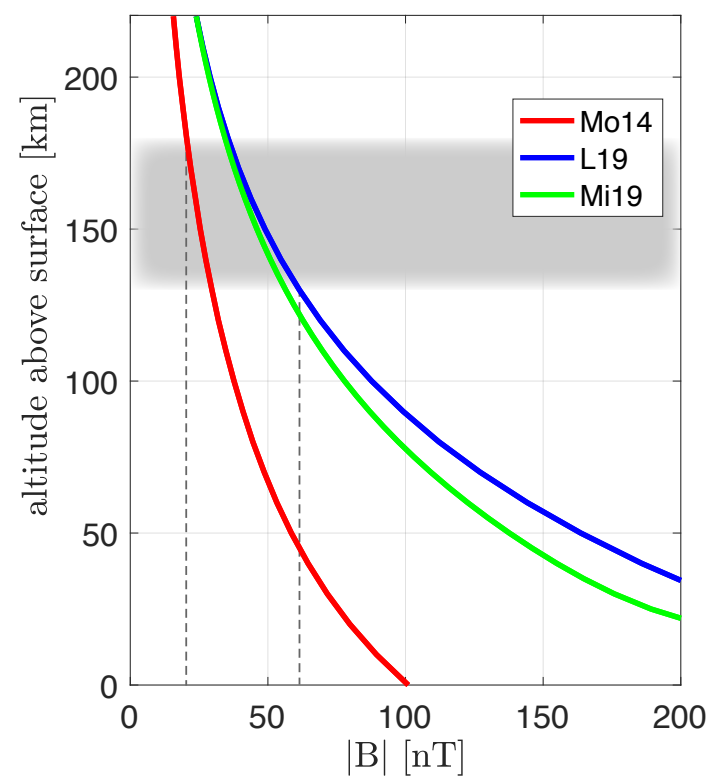

Figure 5. Three different models of the crustal magnetic field predicted at the InSight Landing Site. Mo14 (blue) includes only MGS data (Morschhauser et al., 2014)), the others also include MAVEN data and L19 is a global model (Langlais et al., 2019) and Mi19 is a local model (updated with data up to December 2019 from (Mittelholz et al., 2018)). The grey zone represents the dynamo region.

MAVEN data from November 2014-February 2020 can be used to investigate the residual magnetic field after subtraction of the crustal field (Figure 6). Above weak and strong crustal field regions (Figure 6a and c respectively) external fields show statistically different characteristics during the day. Regions of strong crustal fields show large external fields down to the lowest MAVEN altitudes, whereas weak crustal field regions show lower peak residual fields, up to $\sim 50 \mathrm{nT}$, at $\sim 300 \mathrm{~km}$ altitude. The crustal field above the InSight landing site is substantial, but less than that over e.g. regions of the southern hemisphere (Figure 6 caption), and Figure 6 shows that the day-side residual magnetic fields above InSight (6b) are intermediate between those above weak (6a) and strong (6c) crustal field regions. All regions clearly show a dependence on LTST with little to no external field contributions on the night-side.

The magnetic field residuals in Figure 6 represent the net effect of any external fields, including the draped IMF and any interaction with the crustal field, as well as the ionospheric fields that we are attempting to estimate here. Unmodeled crustal fields are also included, but should be fairly small for shown altitudes because these data directly dictate the model and are not result of downward continuation (Langlais et al., 2019). Furthermore, crustal fields should be invariant with respect to local time; any residual signal should be seen at night and such signals are notably absent in Figure 6. We cannot separate contributions to the residual field above from ionospheric currents and the draped IMF. Furthermore, depending on the orientation and magnitude of the draped IMF relative to the crustal field at a given altitude, the external fields can either enhance or reduce the crustal field. As a result, in what follows we simply use the crustal field strength as background magnetic field strength at dynamo altitudes, but we discuss how this assumption might affect the results in Sections 4.3 and 4.4 . 


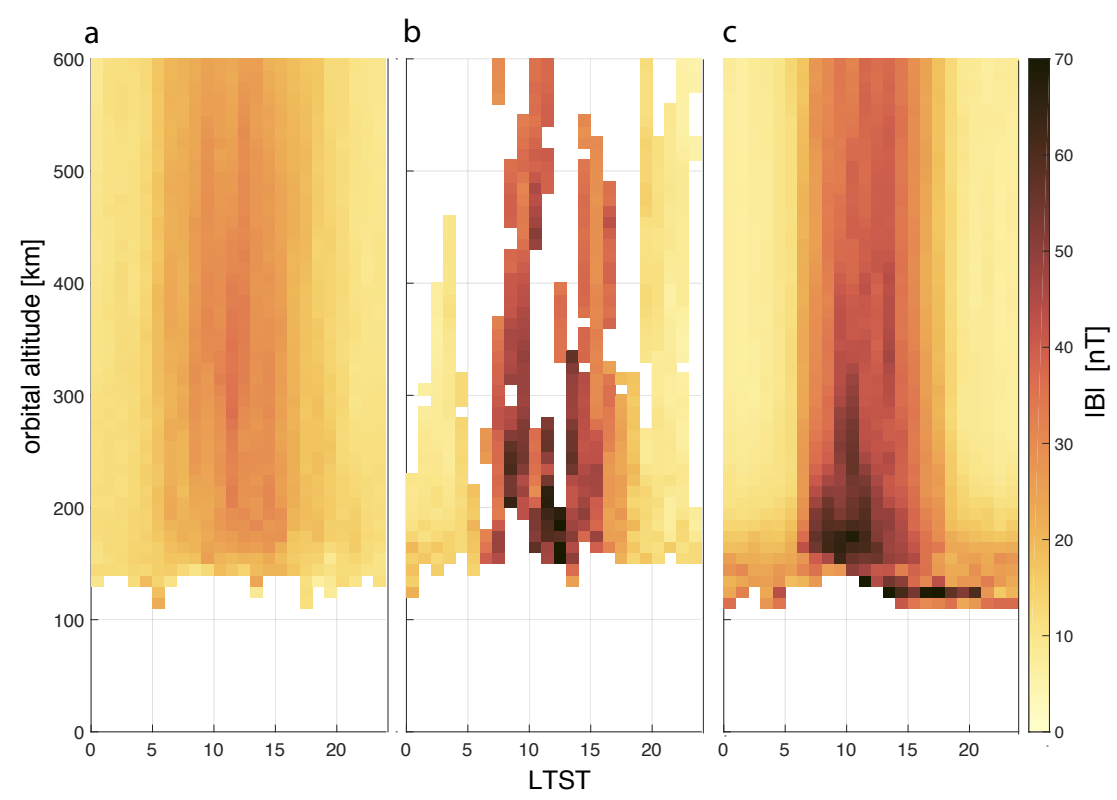

Figure 6. Binned residual amplitudes (data minus the model prediction of L19) above (a) weak crustal magnetization, (b) the InSight landing site, and (c) strong crustal magnetization for bins with more than 50 data points. The data above (a) weak magnetization includes $235^{\circ} \mathrm{E}-285^{\circ} \mathrm{E}$ in the Northern hemisphere (b) the InSight region includes a squared area $10^{\circ}$ in each direction from the Landing Site Coordinates $4.5^{\circ} \mathrm{N}$ and $135.6^{\circ} \mathrm{E}$, (c) strong magnetization includes $135^{\circ} \mathrm{E}-240^{\circ} \mathrm{E}$ and below $-5^{\circ}$ in the Southern hemisphere. The mean and standard deviation crustal field (subtracted and thus not shown in figure) of all data between 140 and $150 \mathrm{~km}$ altitude for the different regions are: (a) $4 \mathrm{nT} \pm 2 \mathrm{nT}$, (b) $29 \mathrm{nT} \pm 23 \mathrm{nT}$, and (c) $230 \mathrm{nT} \pm 168$ nT. 


\subsection{Atmosphere at Ionospheric Altitudes}

MGCMs allow us to build an understanding of the variability of atmospheric conditions that influence the current density, $j$, in the dynamo region. We use the Mars climate database V5.3 (Millour et al., 2017), to produce a set of simulations of the Martian atmosphere using the Laboratoire de Météorologie Dynamique MGCM (Forget et al., 1999). This MGCM computes the seasonal evolution of the large-scale meteorological variables and transported species, from the surface to the exosphere at an altitude of $250 \mathrm{~km}$ (González-Galindo et al., 2013; Chaufray et al., 2015). It is coupled with an ionospheric module that predicts electron density in the upper atmosphere of Mars (GonzálezGalindo et al., 2013).

We first generated a time series of the horizontal winds $\left(v_{\mathrm{H}}\right)$ and electron number density $\left(n_{\mathrm{e}}\right)$ above the location of Insight $\left(4.502^{\circ} \mathrm{N}, 135.623^{\circ} \mathrm{E}\right)$ as a function of LTST every $30^{\circ}$ of solar longitude at an altitude of $130 \mathrm{~km}$, close to the altitude of peak ionization in the atmosphere (Figure $7 \mathrm{a}, \mathrm{b}$ ). These show that $n_{\mathrm{e}}$ is roughly symmetrical about noon, and varies by $25 \%$ over a martian year. In contrast, wind speeds peak in the morning and the amplitude of the peak speed varies by more than a factor of 3 over the year. We approximate the current density at this altitude (assuming that it is within the dynamo region) as $j=n_{\mathrm{e}} q\left(v_{\mathrm{i}}-v_{\mathrm{e}}\right)$ where $n_{\mathrm{e}}$ is electron number density (and ion number density assuming charge neutrality is preserved), $q$ is the elementary charge and $v_{\mathrm{i}}$ and $v_{\mathrm{e}}$ are the speed of ions and electrons respectively. Furthermore, we assume the differential velocity is driven by ions. In reality, electrons drift perpendicular to the neutral wind while they are also pushed in the wind direction by collision with neutrals. At the base of the dynamo region, the net result is that they drift at a $45^{\circ}$ angle to the neutral wind, with a speed of half the neutral wind speed both parallel and perpendicular to the neutral wind. Higher in the dynamo region, the electrons drift at a higher angle to the neutral wind, but as the electron collision frequency decreases, the electron velocity decreases approximately as the ratio of the collision frequency to the gyrofrequency. Thus our assumption that the electrons are stationary overpredicts the magnitude of the current by less than $30 \%$ at the base of the dynamo region. Higher in the dynamo region, this assumption becomes increasingly more valid. Therefore, in the following we assume that electrons are stationary which gives an upper limit on the estimated current and the associated magnetic field.

Ions however are transported by winds, so we use the horizontal wind speed from the MGCM for $v_{\mathrm{i}}$. The vertical wind contribution is minimal, about one order of magnitude weaker (González-Galindo et al., 2009), and can thus be ignored. The LTST at which $j$ peaks lies between $\sim 09.00$ and $\sim 11.00 \mathrm{hr}$ (Figure $7 \mathrm{c}$ ). This peak occurs at $9 \mathrm{am}$ for solar longitudes between $330^{\circ}-360^{\circ}$ and $0^{\circ}-120^{\circ}$. Note that adding dust in the atmosphere (in the MGCM runs) delays the local time of the current peak for solar longitudes $330^{\circ}-360^{\circ}$ by up to 2 hours (not shown here). InSight data show a recurrent dominant peak (Figure $1 \mathrm{~b}-\mathrm{i}$ ) just before $10 \mathrm{am}$ and a second peak after $10 \mathrm{am}$ for earlier sols (Figure $1 \mathrm{c}-\mathrm{d}$ ), which could be driven by dust in the atmosphere. We can however make an important prediction that is confirmed by InSight data: the current density at a given altitude, and hence the resulting magnetic field, peaks in the mid morning.

However, the total ionospheric current depends on the vertically integrated current density in the dynamo region, and requires (a) altitude profiles of $n_{\mathrm{e}}$ and $v_{\mathrm{H}}$ as a function of $L_{\mathrm{S}}(\mathrm{b})$ consideration of changes in these profiles depending on dust conditions in the atmosphere, and (c) estimation of the altitude range spanned by the dynamo region for each of the scenarios in (a) and (b). We thus obtained profiles of $n_{\mathrm{e}}$ and $v_{\mathrm{H}}$ for altitudes between 0 and $220 \mathrm{~km}$ as a function of $L_{\mathrm{S}}$, as well as the neutral density profile for $\mathrm{CO}_{2}$ which is needed for estimating the lowermost altitude of the dynamo region. We used average solar conditions, both with and without a dust storm scenario during dust storm season, $L_{\mathrm{S}}=180^{\circ}-360^{\circ}$ (Figure 8a). Because we are interested in the evo- 
a

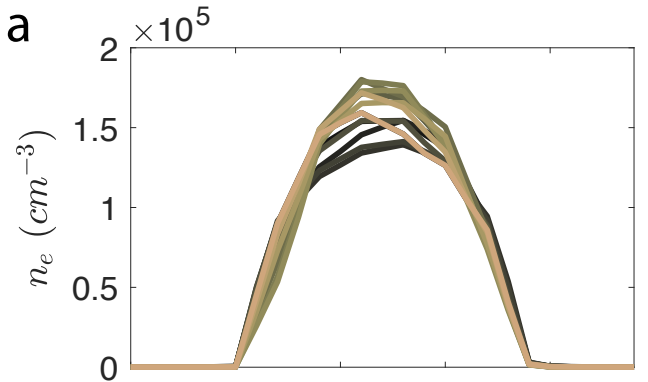

b
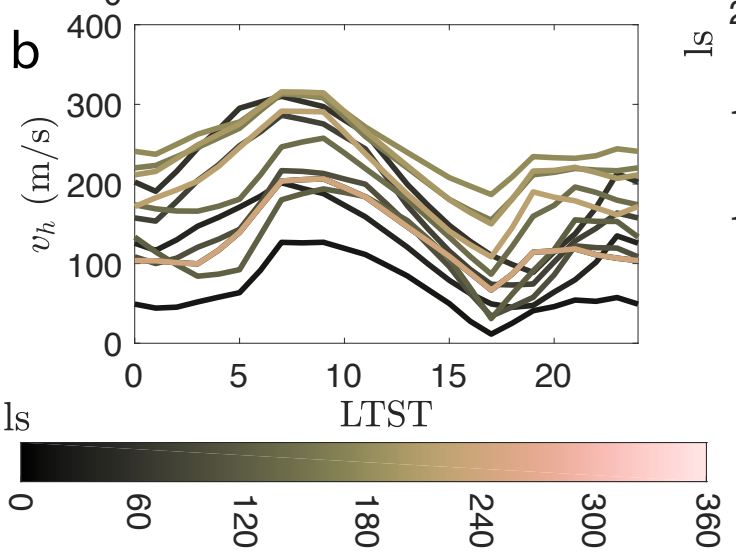

C

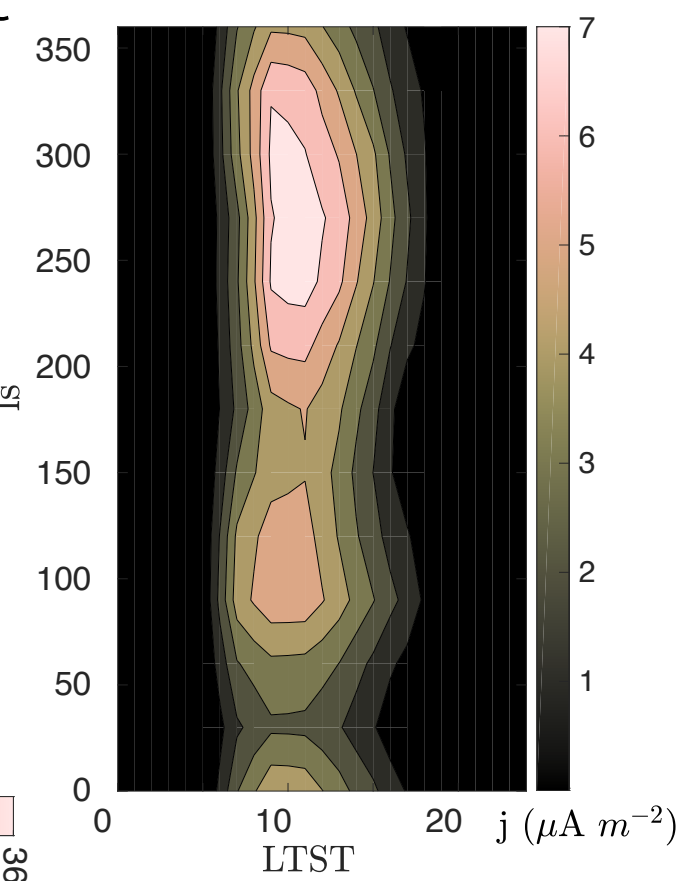

Figure 7. (a) Electron number density, $n_{e}$, and (b) horizontal wind speed, $v_{\mathrm{H}}$, at $130 \mathrm{~km}$ altitude with local time for different solar longitudes (Ls) and (c) the resulting current $j$.

We first calculate current densities assuming that they can be produced at any altitude (i.e., we simply compute $j=n_{\mathrm{e}} q v_{\mathrm{i}}$ ). We assess the actual altitude range of the dynamo region below. The current densities vary with season and the peak amplitudes fluctuate by more than a factor of two, reaching between 2.8 and $7.2 \mu \mathrm{Am}^{-2}$ (Figure 8a). The dust scenario also affects the current density amplitude, which reaches a maximum current density of $8.2 \mu \mathrm{Am}^{-2}$ for $L_{\mathrm{S}}=180^{\circ}$. The main effect of the dust is an increase in the altitude of the peak compared to the no-dust scenario.

\subsection{Estimating the lower boundary of the dynamo region}

Wind-driven magnetic fields can be estimated using the vertically-integrated current density within the dynamo region. The upper boundary of the dynamo region is at $\sim 180 \mathrm{~km}$ altitude, in a region where any current density would be very small (see Figure 8a). However, the lower boundary lies around $130 \mathrm{~km}$, in a region where the current density as calculated above can be large (Figure 8a). Thus the position of this lower boundary is important and can greatly increase or decrease the vertically-integrated current density if it moves down or up respectively. The lower boundary is defined by the altitude at which the electron gyrofrequency, $\omega_{\mathrm{e}}$, and the electron-neutral collision frequency, $\nu_{\mathrm{e}, \mathrm{n}}$, are equal. The background magnetic field modulates $\omega_{\mathrm{e}}$, and the neutral density $n$ and electron temperature $T_{\mathrm{e}}$ modulate $\nu_{\mathrm{e}, \mathrm{n}}$. We use the L19 crustal field model (Langlais et al., 2019) to calculate $\omega_{\mathrm{e}}$ under the assumption that the crustal field is the most important contribution to the ambient magnetic field at $130 \mathrm{~km}$. Doubling the crustal field estimate, which is probably an overestimate of possible external field contributions and unmodeled crustal fields at this altitude (see Figure 6), leads to a change of about $2 \mathrm{~km}$ 
a

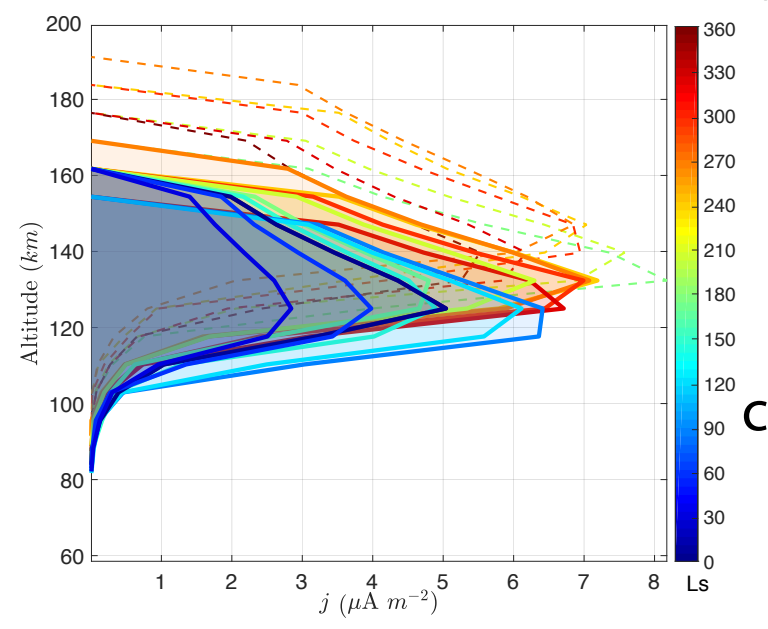

b

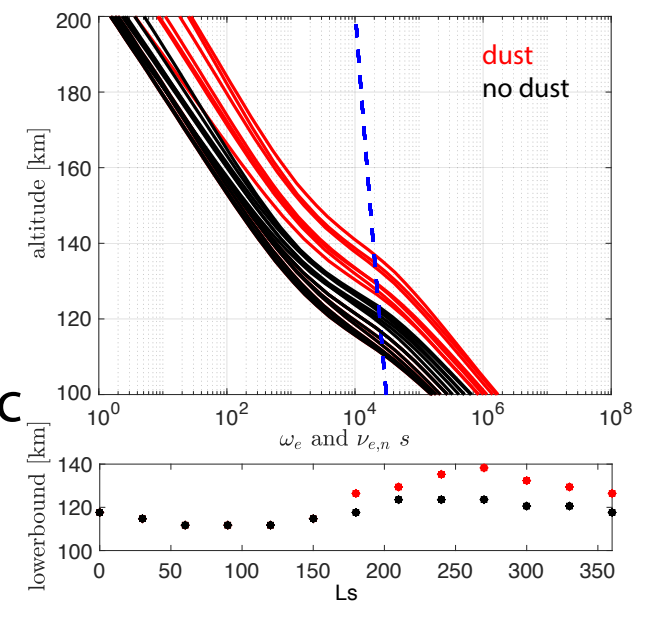

Figure 8. (a) Wind driven current densities for different seasons, initially assuming currents are possible at any altitude. For solar longitude (Ls) 180-360 dashed lines represent a dust storm scenario. (b) Electron gyration frequency, $\omega_{e}$ (blue), and electron-neutral collision frequency, $\nu_{e, n}$, for different Ls (black) and with a dust scenario (red). The lower dynamo boundary is where $\nu_{e, n}=\omega_{e}$ and is shown in (c) as a function of season for the dust-free and dust scenarios.

in the lower boundary and we conclude that sensitivity to the magnetic field is small enough to justify neglecting additional external fields. We further estimate $\nu_{\mathrm{e}, \mathrm{n}}$ under the assumption that $\mathrm{CO}_{2}$ is the dominant neutral constituent below $200 \mathrm{~km}$ (Chen et al., 1978; Schunk \& Nagy, 2009). For $T_{\mathrm{e}}$ we use a functional fit derived from daytime MAVEN Langmuir probe data (Ergun et al., 2015). The resulting lower bound of the dynamo region (Figure 8b) fluctuates with season, from $115 \mathrm{~km}$ to $126 \mathrm{~km}$ altitude and a mean of 121 $\mathrm{km}$, and dust consistently raises the altitude of the boundary up to $141 \mathrm{~km}$.

\subsection{Magnetic fields due to ionospheric currents}

The current density integrated between the lower and upper dynamo boundaries, notated as $\sum j(z)$ in the following, allows us to estimate the magnetic field strength from the wind-driven current system (Figure 9a) at ionospheric altitudes (Withers et al., 2005), $B_{\text {dynamo. }}$ This requires an assumption about the geometry of the current and we calculate two end-members for (i) an infinite current sheet and (ii) a line current. For both of these end-members we can also approximate the resulting field strength at the surface $B_{\text {surface }}$.

For an infinitesimal current sheet, we assume steady-state currents of large extent in horizontal direction with a finite thickness. This case reflects a continuous line of line currents in the ionosphere each determined by the "right-hand-rule" and thus, the field does not change with distance from the sheet, but only changes sign depending on the location above or below the current sheet. The field at the edge of the dynamo region and at the surface are given by $B_{\text {surface }}=B_{\text {dynamo }}=\mu_{0} * \sum j(z) * 1 / 2$.

For a line current, we assume that the width and thickness of the current region are equal resulting in the expression for the field at the edge of the dynamo region, $B_{\text {dynamo }}=$ $\mu_{0} * \sum j(z) * 1 / \pi$. The magnetic field estimate can be scaled to the surface, using the scaling with distance from a line current at the mid-altitude of the dynamo region, as $B_{\text {surface }} / B_{\text {dynamo }}=L /(2 H \pi)$, where $L$ is the extent and $H$ the altitude of the mid- 
point of the dynamo region. These two parameters vary with season, but are about 55 $\mathrm{km}$ for $L$ and $150 \mathrm{~km}$ for $H$.

We calculate the values for $B_{\text {dynamo }}$ and $B_{\text {surface }}$ for the InSight mission so far and projected until the end of the primary mission, i.e., to the end of a martian year. For the current sheet, the predicted magnetic field amplitude at ionospheric altitudes and at the surface are equal and thus the surface prediction gives an upper bound estimate of $B_{\text {surface }}$. This results in a mean and standard deviation of $B_{\text {dynamo }}=B_{\text {surface }}=83$ $\pm 15 \mathrm{nT}$, and $105 \pm 22 \mathrm{nT}$ in a dust scenario. The line current approximation results in a mean and standard deviation magnetic field amplitude of $B_{\text {dynamo }}=53 \pm 10 \mathrm{nT}$ and $67 \pm 16 \mathrm{nT}$ in a dust scenario and $B_{\text {surface }}=9.5 \pm 1.8 \mathrm{nT}$ and $12.1 \pm 2.5 \mathrm{nT}$ in a dust scenario. Dust leads to an increase in magnetic field amplitude because although the altitude of the lower dynamo boundary increases, it is also results in an increase in the altitude of the peak current density. The net result is that the peak current density moves into in the dynamo region. In contrast, for the non-dust scenarios the peak current densities do not always lay in the dynamo region.

These estimates are consistent with previous estimates from MHD simulations (Lillis et al., 2019), and the predicted fields at ionospheric altitudes from satellite data (Figure 6). However, two aspects are important in comparison with Figure 6: (1) The lack of data at dynamo altitudes with peak current densities does not allow for a direct comparison of predicted and observed magnetic field amplitudes. (2) The region shown to represent the external field environment around InSight includes data within $10^{\circ}$ of the landing site (Figure 6b) to increase the number of MAVEN data and even then, there are fewer than 100 points per altitude bin at altitudes below $160 \mathrm{~km}$. The crustal field in this region is quite heterogeneous with field strengths at $130 \mathrm{~km}$ altitude varying by a factor of two (see extended data figure in Johnson et al. (2020) for surface field). This would affect currents produced in the dynamo region and predictions in this study are limited to the crustal field right above the InSight landing site.

We compare the variability observed in the InSight magnetic field data (Figure 9) with our estimates for wind-driven ionospheric fields. Such fields for the 26-day median sols are $20-40 \mathrm{nT}$ peaking during the dust storm and lying between our two end-member scenarios for the wind-driven estimates. An increase in the daily peak amplitude is seen in InSight data at the time of the regional dust storm, with peak values 10-20 nT larger than at subsequent times. The wind-driven currents predict a steady increase in the peak field from InSight Sol 180 to Sol 300 that is not seen in the IFG data. Regional or global dust storms in the upcoming dust season will be of particular interest because larger amplitude fields are predicted during those times. Sol-to-sol variability in the peak fields observed by InSight (Figure 2b) likely reflects local changes in ionospheric currents that are not captured in our simple, slowly-time-varying model. 


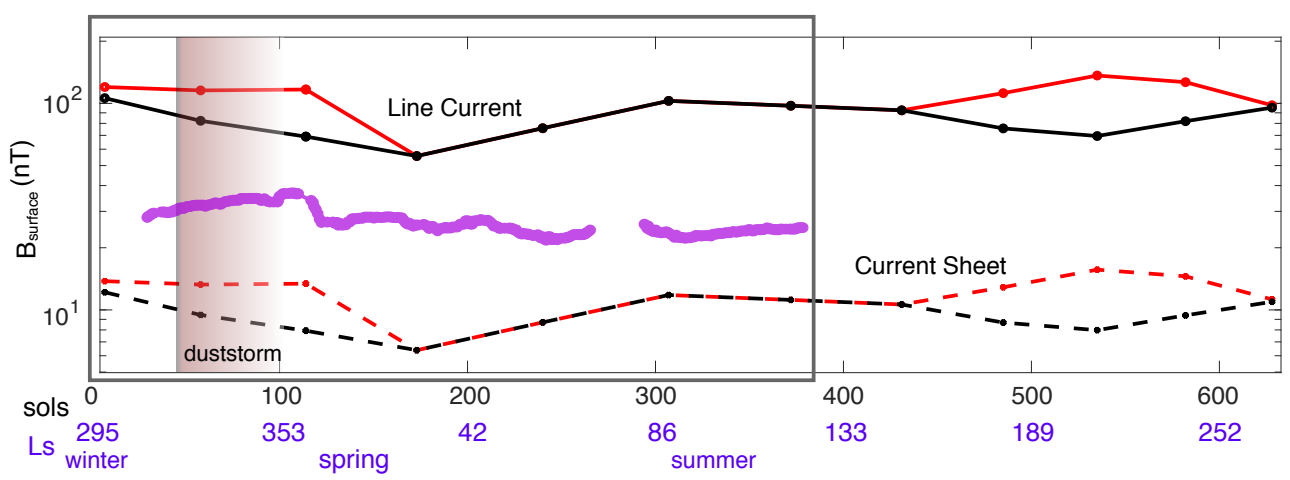

Figure 9. Wind driven magnetic field response, $|B|$ at the surface assuming that the dynamo region is a line current (solid line) or a current sheet (dashed line). The black line shows an average scenario, the red line shows a dust storm scenario during dust season. The brown area highlights the time at which a dust storm actually occurred during the InSight mission. The curve from Figure 3b shows the maximum amplitude of the observed magnetic field in a 26-sol running mean (purple) for easier comparison with wind-driven predictions.

Finally, we also note that the vertically-integrated currents depend strongly on the location of the lower dynamo boundary, and this in turn is affected by the background magnetic field. For example, a weaker background field such as the region shown in Figure $6 \mathrm{a}$ would lead to an increased altitude of the lower boundary and thus a smaller amplitude vertically-integrated current in the dynamo region and weaker resulting fields. Similarly a stronger background field, which in the regions of strong crustal fields can be several hundred $\mathrm{nT}$ at ionospheric altitudes and sometimes over $1000 \mathrm{nT}$, could decrease the altitude of the lower boundary resulting in a larger amplitude vertically-integrated current in the dynamo region and stronger resulting fields. Taking 0.1 and 10 times the crustal field magnitude from the InSight landing site result in lower dynamo boundary altitudes from $129-141 \mathrm{~km}$ and from $100-112 \mathrm{~km}$ respectively. This could contribute, at least in part, to explaining the differences in the residual fields observed by MAVEN on the dayside at ionospheric altitudes over weak and strong field regions (Fig 6a and c) compared with those observed over the InSight region. Thus, we would expect to qualitatively observe a similar pattern at a different landing site, but different amplitudes of such signals. This might be of interest in future mission planning in which external magnetic fields are of interest. This includes future electrical conductivity studies in which external fields are used as inducing source fields.

\section{Conclusions}

Magnetic field data recorded by the IFG over the first 389 sols of the InSight mission reveal daily variations in the magnetic field with typical peak amplitudes of 20-40 $\mathrm{nT}$. The peak amplitude fields usually occur in the early- to mid-morning. Larger amplitude fields were observed during the first $~ 100$ sols. Sol-to-sol variability in peak amplitudes can be large, and the peak field at the surface to date, unassociated with lander activities is $\sim 80 \mathrm{nT}$. Assessment of the calibration procedure for IFG data, indicate that temperature and solar array current variations have minimal influence on the final calibrated data product. The largest uncertainty in any such remaining contributions is the effect of solar array currents. This results from the lack of information on the geometry of the solar array currents at any given time and the resulting magnetic fields that would be expected at the IFG. We also note that solar array current data at a 1 $\mathrm{Hz}$ frequency whenever the lander is on has recently (since sol 426, February 7, 2020) 
become available, which will be of benefit to future calibration efforts. Lander activities have clear signals, that cannot be completely removed, but these are intermittent and occur on time scales that are short compared to the daily variations of interest here. Winddriven ionospheric currents produce magnetic fields; these depend on atmospheric properties such as horizontal wind speed and electron density that we model with a Mars Global Circulation Model. We compare this "average" state with that during a dust storm scenario, and compare the resulting magnetic field predictions with data observed during the first 389 InSight sols. This simple model predicts the observed magnetic field amplitude to within an order of magnitude. Furthermore, the predicted increase in magnetic field amplitude of up to a factor of two during a dust storm scenario can be identified in the data. We conclude that wind-driven currents are a major contributor to the diurnal signal seen at the surface. These results indicate that ionospheric currents on Mars provide a daily time-varying source field that could be used to probe the electrical conductivity structure of the martian mantle.

Data analyzed in this study comprise just over half a year (0.58 Mars years) on Mars. Continued operation of InSight on Mars would allow future studies to investigate seasonal variability in greater detail, and in particular elucidate the effects of regional versus global dust storms. It will also allow investigations of modulation of the diurnal signal at periods such as the 26-day cycle associated with the IMF. Although the time frame we discuss covers 15 such cycles, multiple PAEs early in the mission and a communication issue during solar conjunction interrupt the existing time series, making such analyses difficult. However, even visual inspection of the data collected so far indicate that any 26-day signal (or harmonics thereof), if present, are smaller in amplitude than the typical sol-to-sol variability in the diurnal magnetic field signal. Finally, the time-varying magnetic fields reported here provide steps toward characterizing the surface electromagnetic environment on Mars, specifically during the minimum between solar cycles 24 and 25. Changes in the surface magnetic field during the ascending phase of solar cycle 25 , in particular the effects of space weather, will be of particular interest and greatly facilitated by joint surface (InSight) and satellite (MAVEN) observations.

\section{Acknowledgments}

We acknowledge NASA, CNES, their partner agencies and Institutions (UKSA, SSO, DLR, JPL, IPGP-CNRS, ETHZ, IC, MPS-MPG) and flight operations team at JPL, SISMOC (SEIS on Mars Operations Center) and MSDS (Mars SEIS Data Service). We acknowledge support from the Natural Sciences and Engineering Research Council of Canada, the Canadian Space Agency and the InSight Mission (A.M. and C.L.J.), as well as CNES in the frame of the InSight mission (A.S, F.F., B.L.) and NASA Award 80NSSC18K1632 (M.F.). We thank Fabian Euchner and John Clinton from the Marsquake Service for compiling lander activity events and Francisco Gonzalez-Galindo for his contributions to the development of the LMD GCM Ionosphere model. We also thank David Brain and Günther Kletetschka for thoughtful reviews that improved the manuscript. All MAVEN and InSight data used in this study are publicly available in the Planetary Data System: InSight IFG calibrated data https://doi.org/10.17189/1519202 and Calibration data https://doi.org/10.17189/1510486, and MAVEN calibrated MAG data urn:nasa: pds:maven.mag.calibrated::2.12. All derived data products are available and hosted at https://doi.org/10.6084/m9.figshare.12564191. This paper is InSight Contribution Number 167.

\section{References}

Banerdt, W. B., Smrekar, S., Banfield, D., Giardini, D., Golombek, M. P., Johnson, C., ... Wieczoreck, M. (2020). Initial Results from the InSight Mission on Mars. Nature Geoscience, 13(March), 183-189. Retrieved from http:// dx.doi.org/10.1038/s41561-020-0544-y doi: 10.1038/s41561-020-0544-y 
Banfield, D., Rodriguez-Manfredi, J. A., Russell, C. T., Rowe, K. M., Leneman, D., Lai, H. R., ... Team, T. T. (2018). InSight Auxiliary Payload Sensor Suite (APSS). Space Science Reviews, 215(1), 4. Retrieved from https://doi.org/ 10.1007/s11214-018-0570-x doi: 10.1007/s11214-018-0570-x

Chaufray, J. Y., Gonzalez-Galindo, F., Forget, F., Lopez-Valverde, M. A., Leblanc, F., Modolo, R., \& Hess, S. (2015). Variability of the hydrogen in the martian upper atmosphere as simulated by a 3D atmosphere-exosphere coupling. Icarus, 245(0), 282-294. Retrieved from http://www.sciencedirect .com/science/article/pii/S0019103514004540\{\\%\}5CnUsers/wang/ GoogleDrive/Papers/chaufray2015.pdf doi: 10.1016/j.icarus.2014.08.038

Chen, R. H., Cravens, T. E., \& Nagy, A. F. (1978). The Martian ionosphere in light of the Viking observations. Journal of Geophysical Research: Space Physics, 83(A8), 3871-3876. Retrieved from https://agupubs.onlinelibrary.wiley .com/doi/abs/10.1029/JA083iA08p03871 doi: 10.1029/JA083iA08p03871

Chi, P., Russell, C., Yu, Y., Joy, S., Ma, Y., Banfield, D., ... Banerdt, W. $\quad$ (2019, dec). InSight Observations of Magnetic Pulsations on Martian Surface: Morphology and Wave Sources. In Agu fall meeting abstracts (Vol. 2019, pp. DI51B-0024).

Ergun, R. E., Morooka, M. W., Andersson, L. A., Fowler, C. M., Delory, G. T., Andrews, D. J., ... Jakosky, B. M. (2015). Dayside electron temperature and density profiles at Mars: First results from the MAVEN Langmuir probe and waves instrument. Geophysical Research Letters, 42(21), 8846-8853. doi: 10.1002/2015GL065280

Fillingim, M. O., Lillis, R. J., England, S. L., Peticolas, L. M., Brain, D. A., Halekas, J. S., .. Bougher, S. W. (2012). On wind-driven electrojets at magnetic cusps in the nightside ionosphere of Mars. Earth, Planets and Space, 64 (2), 93-103. doi: 10.5047/eps.2011.04.010

Fillingim, M. O., Peticolas, L. M., Lillis, R. J., Brain, D. A., Halekas, J. S., Lummerzheim, D., \& Bougher, S. W. (2010). Localized ionization patches in the nighttime ionosphere of Mars and their electrodynamic consequences. Icarus, 206(1), 112-119. Retrieved from http://dx.doi.org/10.1016/ j.icarus.2009.03.005 doi: 10.1016/j.icarus.2009.03.005

Forget, F., Hourdin, F., Fournier, R., Hourdin, C., Talagrand, O., Collins, M., ... Huot, J. P. (1999). Improved general circulation models of the Martian atmosphere from the surface to above $80 \mathrm{~km}$. Journal of Geophysical Research: Planets, 104(E10), 24155-24175. doi: 10.1029/1999JE001025

González-Galindo, F., Chaufray, J.-Y., López-Valverde, M. A., Gilli, G., Forget, F., Leblanc, F., ... Yagi, M. (2013). Three-dimensional Martian ionosphere model: I. The photochemical ionosphere below $180 \mathrm{~km}$. Journal of Geophysical Research: Planets, 118(10), 2105-2123. Retrieved from https://agupubs.onlinelibrary.wiley.com/doi/abs/10.1002/jgre. 20150 doi: $10.1002 /$ jgre.20150

González-Galindo, F., Forget, F., López-Valverde, M. A., i Coll, M., \& Millour, E. (2009). A ground-to-exosphere Martian general circulation model: 1. Seasonal, diurnal, and solar cycle variation of thermospheric temperatures. Journal of Geophysical Research: Planets, 114(E4). Retrieved from https:// agupubs.onlinelibrary.wiley.com/doi/abs/10.1029/2008JE003246 doi: 10.1029/2008JE003246

Jakosky, B. M., Lin, R. P., Grebowsky, J. M., Luhmann, J. G., Mitchell, D. F., Beutelschies, G., ... Zurek, R. (2015). The Mars Atmosphere and Volatile Evolution (MAVEN) Mission. Space Science Reviews, 3-48. Retrieved from http://dx.doi.org/10.1007/s11214-015-0139-x doi: 10.1007/s11214-015-0139-x

Johnson, C. L., Mittelholz, A., Langlais, B., Russell, C. T., Ansan, V., Banfield, D., ... Banerdt, W. B. (2020). Crustal and time-varying magnetic fields 
at the InSight landing site on Mars. N Nature Geoscience, 13(3), 199-204.

Retrieved from http://dx.doi.org/10.1038/s41561-020-0537-x doi: 10.1038/s41561-020-0537-x

Joy, S. P., Mafi, J. N., \& Slavney, S. (2019). Interior Exploration Using Seismic Investigations, Geodesy, and Heat Transport (InSight) Mission Insight Fluxgate Magnetometer (IFG) PDS Archive Software Interface Specification. urn:nasa:pds:insight-ifg-mars:document:insight-ifg-sis.

Langlais, B., Civet, F., \& Thébault, E. (2017). In situ and remote characterization of the external field temporal variations at Mars. Journal of Geophysical Research: Planets, 1-14. Retrieved from http://doi.wiley.com/10.1002/ 2016JE005060 doi: 10.1002/2016JE005060

Langlais, B., Thébault, E., Houliez, A., Purucker, M. E., \& Lillis, R. J. A New Model of the Crustal Magnetic Field of Mars Using MGS and MAVEN. Journal of Geophysical Research: Planets, 124(6), 1542-1569. doi: 10.1029/2018JE005854

Lillis, R. J., Fillingim, M. O., Ma, Y., Gonzalez-Galindo, F., Forget, F., Johnson, C. L., ... Fowler, C. M. (2019). Modeling Wind-Driven Ionospheric Dynamo Currents at Mars: Expectations for InSight Magnetic Field Measurements. Geophysical Research Letters, 46(10), 5083-5091. doi: 10.1029/2019GL082536

Lorenz, R. D., Lemmon, M. T., Maki, J., Banfield, D., Spiga, A., Charalambous, C., ... Banerdt, W. B. (2020). Scientific Observations With the InSight Solar Arrays: Dust, Clouds, and Eclipses on Mars. Earth and Space Science, 7(5), 1-12. doi: 10.1029/2019EA000992

Marquette, M. L., Lillis, R. J., Halekas, J. S., Luhmann, J. G., Gruesbeck, J. R., \& Espley, J. R. (2018). Autocorrelation Study of Solar Wind Plasma and IMF Properties as Measured by the MAVEN Spacecraft. Journal of Geophysical Research: Space Physics, 123, 2493-2512. doi: 10.1002/2018JA025209

Miles, D. M., Mann, I. R., Kale, A., Milling, D. K., Narod, B. B., Bennest, J. R., ... Unsworth, M. J. (2017). The effect of winding and core support material on the thermal gain dependence of a fluxgate magnetometer sensor. Geoscientific Instrumentation, Methods and Data Systems, 6(2), 377-396. Retrieved from https://gi.copernicus.org/articles/6/377/2017/ doi: 10.5194/gi-6-377-2017

Millour, E., Forget, F., Spiga, A., Vals, M., Zakharov, V., Navarro, T., ... MCD/GCM Development Team (2017, apr). The Mars Climate Database (MCD version 5.3). In Egu general assembly conference abstracts (p. 12247).

Mittelholz, A., Johnson, C. L., \& Lillis, R. J. (2017). Global-scale External Magnetic Fields at Mars Measured at Satellite Altitude. Journal of Geophysical Research: Planets, 122(6), 1243-1257. Retrieved from http://doi.wiley.com/ 10.1002/2017JE005308 doi: 10.1002/2017JE005308

Mittelholz, A., Johnson, C. L., \& Morschhauser, A. (2018). A New Magnetic Field Activity Proxy for Mars From MAVEN Data. Geophysical Research Letters, 45(12), 5899-5907. Retrieved from https://agupubs.onlinelibrary.wiley .com/doi/abs/10.1029/2018GL078425 doi: 10.1029/2018GL078425

Morschhauser, A., Lesur, V., \& Grott, M. (2014). A spherical harmonic model of the lithospheric magnetic field of Mars. Journal of Geophysical Research: Planets, 119(6), 1162-1188. Retrieved from http://doi.wiley.com/ 10.1002/2013JE004555\{\\%\}5CnUsers/wang/Downloads/391168.pdf doi: 10.1002/2013JE004555

Opgenoorth, H. J., Dhillon, R. S., Rosenqvist, L., Lester, M., Edberg, N. J., Milan, S. E., ... Brain, D. (2010). Day-side ionospheric conductivities at Mars. Planetary and Space Science, 58(10), 1139-1151. Retrieved from http:// dx.doi.org/10.1016/j.pss.2010.04.004 doi: 10.1016/j.pss.2010.04.004

Riousset, J. A., Paty, C. S., Lillis, R. J., Fillingim, M. O., England, S. L., Withers, 
P. G., \& Hale, J. P. M. (2014). Electrodynamics of the martian dynamo region near magnetic cusps and loops. Geophysical Research Letters, 41(4), 1119-1125. doi: 10.1002/2013gl059130

Rochette, P., Gattacceca, J., Chevrier, V., Hoffmann, V., Lorand, J. P., Funaki, M., \& Hochleitner, R. (2005, apr). Matching Martian crustal magnetization and magnetic properties of Martian meteorites. Meteoritics and Planetary Science, 40, 529. doi: 10.1111/j.1945-5100.2005.tb00961.x

Schunk, R. W., \& Nagy, A. F. (2009). Electron temperatures in the F region of the ionosphere: Theory and observation. Cambridge university press.

Smrekar, S. E., Lognonné, P., Spohn, T., Banerdt, W. B., Breuer, D., Christensen, U., ... Wieczorek, M. (2018, dec). Pre-mission InSights on the Interior of Mars. Space Science Reviews, 215(1), 3. Retrieved from https://doi.org/ 10.1007/s11214-018-0563-9 doi: 10.1007/s11214-018-0563-9

Thorne, S. N., Mittelholz, A., Johnson, C. L., Joy, S., Liu, X., Russell, C. T., ... Banerdt, W. B. (2020). InSIght fluxgate magnetometer data calibration assessment and implications. LPI Contributions 2089.

Withers, P., Mendillo, M., Rishbeth, H., Hinson, D. P., \& Arkani-Hamed, J. (2005). Ionospheric characteristics above Martian crustal magnetic anomalies. Geophysical Research Letters, 32(16), L16204-L16204. Retrieved from http://dx.doi.org/10.1029/2005GL023483\{\\%\}5CnUsers/wang/ GoogleDrive/Papers/withers2005.pdf doi: 10.1029/2005GL023483

Withers, P., Vogt, M., Mahaffy, P., Benna, M., Elrod, M., \& Jakosky, B. (2015). Changes in the thermosphere and ionosphere of Mars from Viking to MAVEN. Geophysical Research Letters, 42, 1-9. doi: 10.1002/2015GL065985.Abstract

Zou, H., Lillis, R. J., Wang, J. S., \& Nielsen, E. (2011). Determination of seasonal variations in the Martian neutral atmosphere from observations of ionospheric peak height. Journal of Geophysical Research: Planets, 116(9), 1-14. doi: 10.1029/2011JE003833 